\title{
Spatially resolving the wind and disk structures around active B-type stars
}

\author{
Christopher Tycner
}

Department of Physics, Central Michigan University, Mount Pleasant, MI 48859, USA

\begin{abstract}
Long-baseline optical and IR interferometers now routinely resolve the wind and disklike structures around early-type stars. The typical angular scales resolved by current generation of instruments are well bellow the milli-arcsecond level. These type of observations allow, in some cases for the first time, placing very tight constraints on current theories and models of the circumstellar structures around these type of stars. Specific examples of observations obtained at the Navy Prototype Optical interferometer of the spatially resolved regions around a luminous blue variable star P Cyg and a B-type star with circumstellar disk are presented. The need for connection between interferometric observables and physical parameters predicted by theory and numerical models are emphasized.
\end{abstract}

Keywords. techniques: interferometric, stars: emission-line, Be

\section{Introduction}

Interferometric observations of line-emitting early-type stars have shown to be invaluable in constraining the circumstellar regions associated with these type of stars. Most notably the $\mathrm{H} \alpha$ emission line, which happens to be one of the strongest emission lines, provides some of the strongest interferometric signals of the line-emitting regions (see, for example, Stee et al. (1995), Quirrenbach et al. (1997), Tycner et al. (2006)). Although some of the first studies concentrated on spatially resolving the line-emitting regions and have successfully constrained their geometries, it is now possible to directly compare the interferometric observables to detailed numerical disk models.

\section{Methodology}

To go beyond simple geometrical models representing the line-emitting regions around early-type stars, one can utilize numerical disk modeling routines that not only can solve for self-consistent temperature structure by balancing all the heating and cooling mechanisms within the disk, but can provide model interferometric observables that can be directly compared to observations.

To illustrate this strategy we utilize the BEDISK code of Sigut \& Jones (2007). BEDISK computes a non-LTE radiative equilibrium model with solar chemical composition for the circumstellar gas. The numerical model provides monochromatic images of the circumstellar disk over a specific range of wavelengths. This includes the regions associated with the $\mathrm{H} \alpha$ emission line, which is of particular interest if models are to be compared to interferometric observations obtained over that line. Figure 1 illustrates the typical density structure that is used as a starting point and is described by a simple two-component parameterization of the form:

$$
\rho(R, Z)=\rho_{0}\left(\frac{R_{\star}}{R}\right)^{n} e^{-\left(\frac{Z}{H}\right)^{2}},
$$



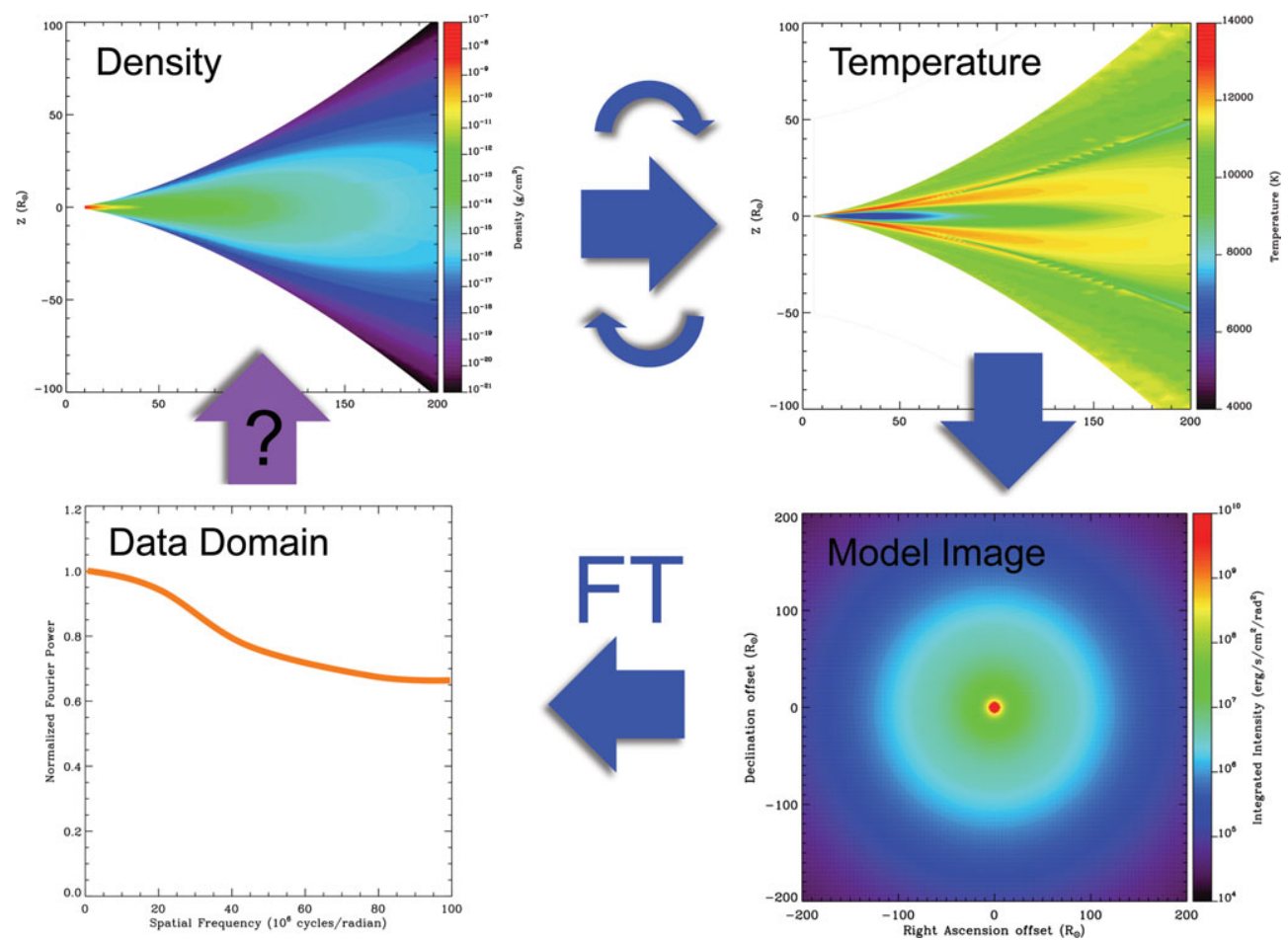

Figure 1. Schematic representation of a typical data flow involved in a comparison of numerical disk model to interferometric observations. A density structure (upper-left) is selected, which in turn is used to calculate (iteratively) a self-consistent temperature structure (upper-right). Once a final density and temperature structure is obtained, a synthetic image in the desired spectral bandpass can be obtained (lower-right). Finally, a Fourier transform of the synthetic image provides model values that can be compared directly to the interferometric observables (lower-left). The entire process can (if desired) be repeated in an iterative sequence.

where $\rho_{0}$ is the density at the inner edge of the disk in the equatorial plane, $n$ is the index in the radial power-law, $H$ is the scale height in the $Z$-direction perpendicular to the plane of the disk, and $R_{\star}$ is the stellar radius (see Sigut \& Jones (2007) for more details). The vertical density structure and the temperature distribution throughout the disk are iterated until all the heating and cooling mechanisms are balanced. The final density and temperature structure is then used to calculate a two-dimensional model image over a wavelength range of interest. Because long-baseline interferometric observations represent the Fourier transform of the source structure on the sky, the final step involves the Fourier transform of the synthetic image. Only then the model can be compared directly to the interferometric observables.

In practice the entire data flow (as illustrated in Figure 1) is either, repeated in an iterative sequence so that a best-fit numerical disk model is found, or as it was demonstrated by Tycner et al. (2008) an extensive grid of models is compared to interferometric observations. The latter approach allows not only a selection of 'best' model, but also testing for any inherent correlations between the free model parameters, such as $\rho_{0}$ and $n$. 


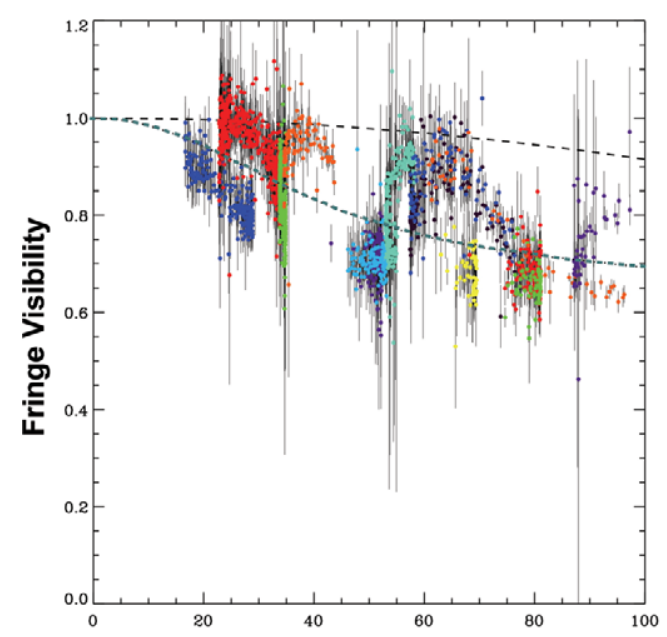

Radial Spatial Frequency $\left(10^{6}\right.$ cycles/rad)

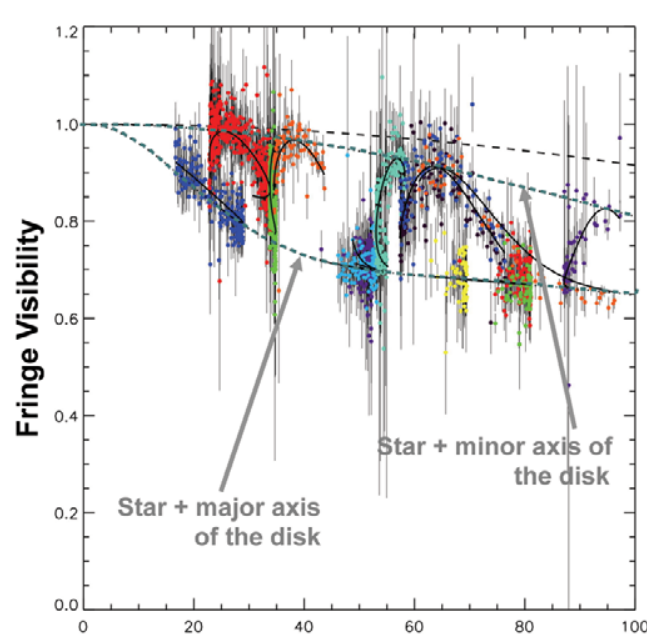

Radial Spatial Frequency $\left(10^{6}\right.$ cycles/rad)

Figure 2. Square visibility data acquired in the $\mathrm{H} \alpha$ channel for the Be $\operatorname{star} \zeta$ Tau using 15 unique baselines (shown in different colors). Both, circularly symmetric (left panel) and elliptical (right panel) Gaussian models are shown. In the case of the elliptical model the extreme model values at minor and major axes are shown (dashed-dotted lines). The mostly unresolved central star represented by uniform disk model is also shown (dashed line).

\section{Be Star}

The Navy Prototype Optical Interferometer (NPOI) operates over a spectral range between 560 and $870 \mathrm{~nm}$. The interferometric signal (which consists of squared visibility measures and sometimes closure phases) is dispersed over 15 spectral channels. Therefore, interferometric observations of $\mathrm{H} \alpha$-emitting sources contain all the line emission in one spectral channel. Figure 2 illustrates a typical signature seen for Be stars that do not possess circularly symmetric disk emission as seen on the sky. Most of this type of asymmetry can be attributed to a simple geometrical projection of how the circumstellar disk is viewed by the observer (i.e., the inclination angle with respect to the plane of the disk).

It is evident from Figure 2 that not only the $\mathrm{H} \alpha$ region is fully resolved, but that it cannot be represented by models that have circular symmetry. Because most, it not all, of such asymmetry can be attributed to a simple projection angle, that means that such data can be compared to circularly symmetric numerical disk models as long as the projection angle is properly accounted for. In the case of the BEDISK numerical routine, this is possible with a ray tracing routine known as BERAY (see contribution by Sigut, this volume).

\section{LBV Star}

In addition to resolving $\mathrm{H} \alpha$-emitting circumstellar structures around B-type stars, one can utilize the same technique to resolve the wind structure around an LBV star. Figure 3 shows the interferometric data from Balan et al. (2010) acquired in the spectral channel containing the $\mathrm{H} \alpha$ emission line of the LBV star P Cyg. The wind structure is fully resolved and data at long enough baselines (i.e., high enough Fourier frequencies) 


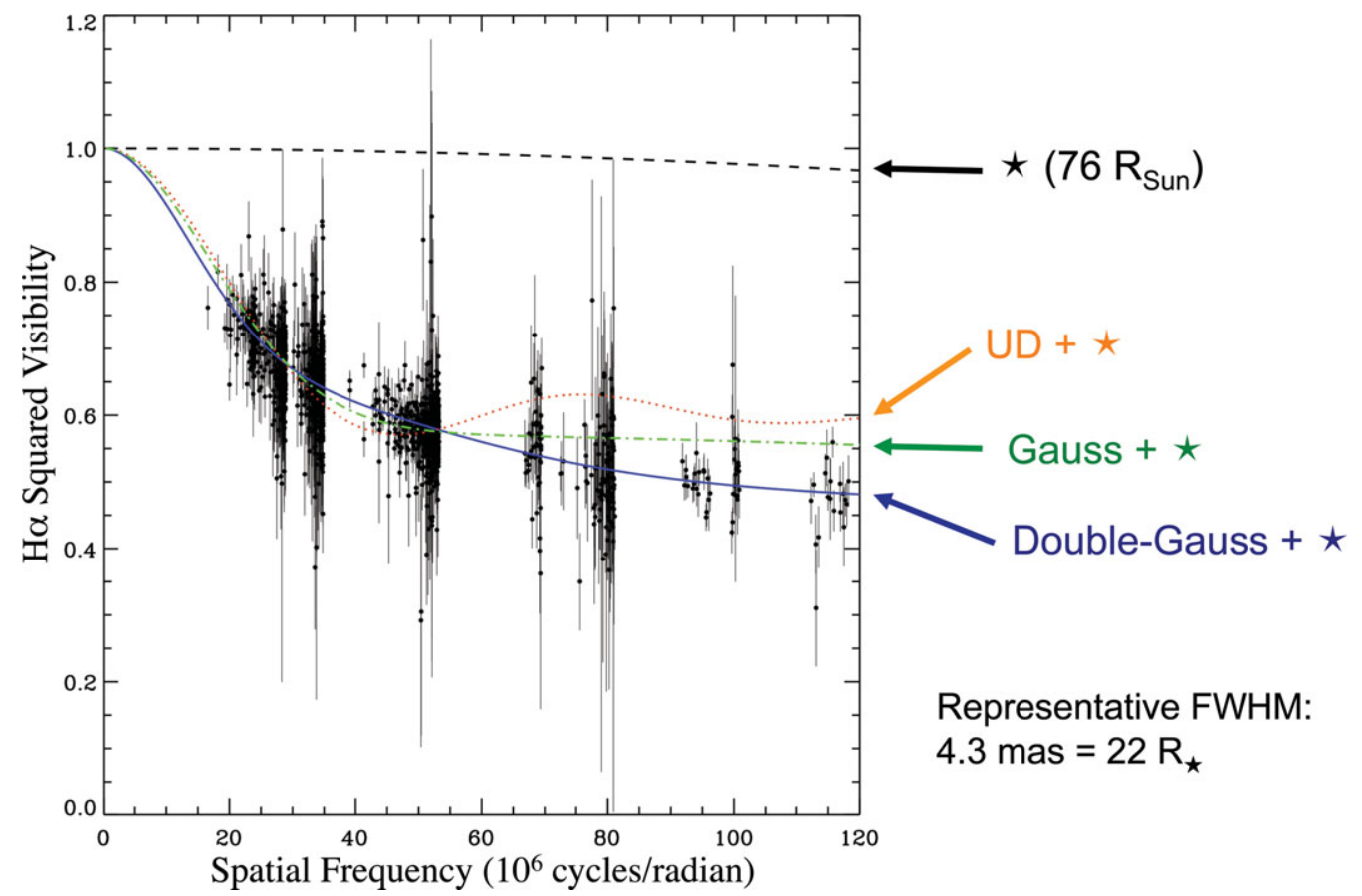

Figure 3. Squared visibility data acquired in the $\mathrm{H} \alpha$ channel of $\mathrm{P}$ Cyg. The model representing the central star (dashed-line) is shown along with three possible models representing the radial fall-off of the $\mathrm{H} \alpha$ emission from the wind structure.

has been acquired that uniform disk (UD) or Gaussian radial intensity distributions can be excluded. The data presented in Figure 3 has been modeled with a two-component Gaussian model, however this only represents a simple geometric parameterization.

Describing the radial intensity distribution around P Cyg with only a few parameters, such as provided by a double-Gaussian model, can be useful when simplicity is desired. However, a much more complete analysis could be accomplished if such interferometric data would be compared directly to model predicted observables. Furthermore, if various competing models describing the wind structure would publish observables that could be either directly, or after an appropriate transform, be compared to interferometric observables, such observations would provide yet another critical test and constraint for such models.

\section{Conclusions}

We have demonstrated how interferometric data for circumstellar disks and wind structures can be directly used to constrain the functional form of the radial intensity distribution on the sky. Although this can provide angular measurements of such regions with unprecedented accuracy, what is needed now is a systematic way of comparing such observations to theoretical models. This could be easily accomplished if analytical and numerical models published in the literature would accompany their predictions with both one- and two-dimensional observables. For example, publishing synthetic images (preferably in digital format) as illustrated in Figure 4 would allow for a direct comparison to data acquired via interferometry and possibly a combination of observational methods. 

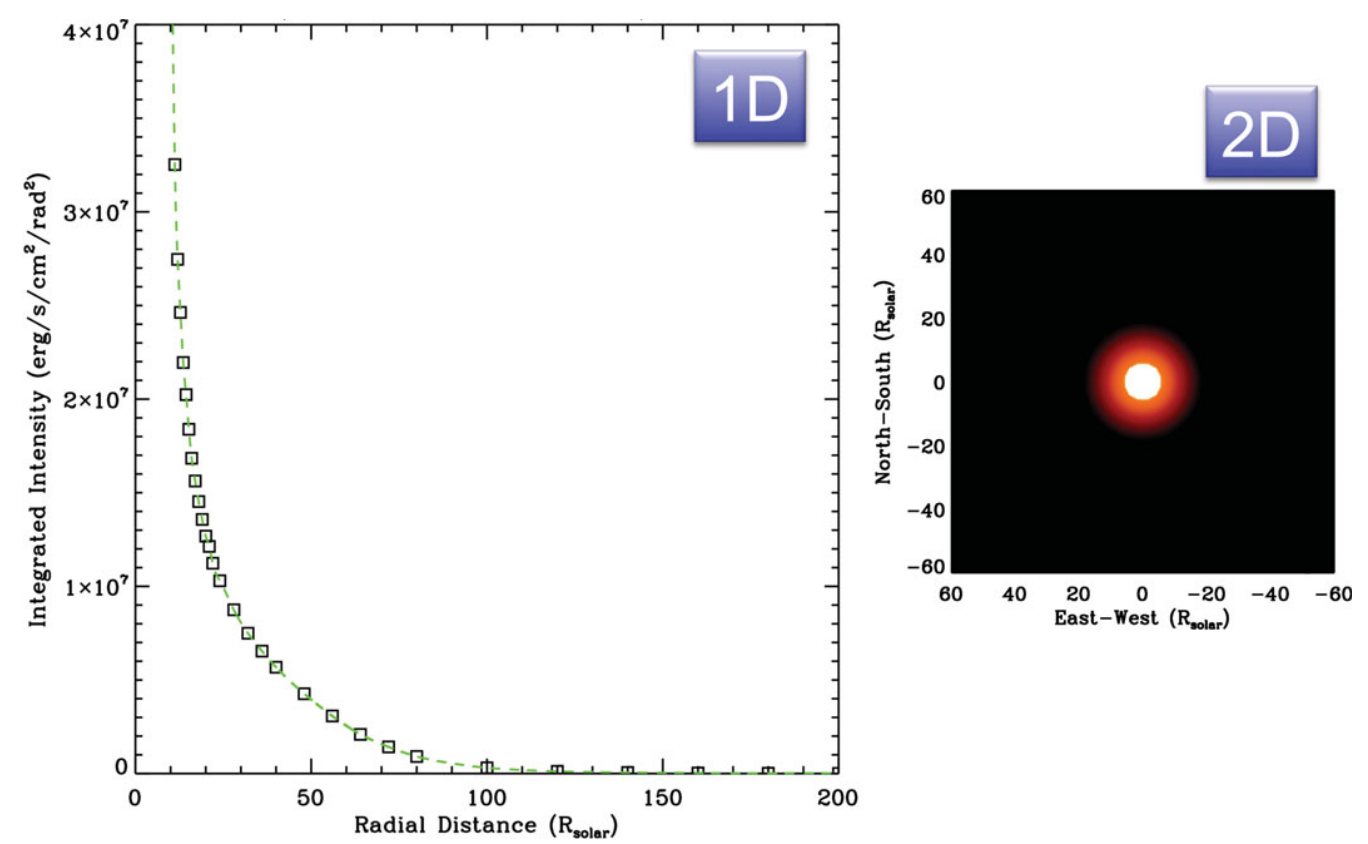

Figure 4. Integrated intensity of a synthetic model as a function of radial distance from the central star over a spectral channel containing an $\mathrm{H} \alpha$ emission line. Both, radial profile of the intensity distribution (left panel) and a 2-D contour plot of the intensity (right panel) are shown.

\section{Acknowledgements}

The Navy Prototype Optical Interferometer is a joint project of the Naval Research Laboratory and the US Naval Observatory, in cooperation with Lowell Observatory, and is funded by the Office of Naval Research and the Oceanographer of the Navy. C.T. would also like to acknowledge, with thanks, financial support from the Central Michigan University.

\section{References}

Balan, A., Tycner, C., Zavala, R. T., Benson, J. A. et al. 2010, AJ, 139, 2269

Quirrenbach, A., Bjorkman, K. S., Bjorkman, J. E., Hummel, C. A. et al. 1997, ApJ, 479, 477

Sigut, T. A. A. \& Jones, C. E. 2007, ApJ, 668, 481

Stee, P., de Araujo, F. X., Vakili, F., Mourard, D. et al. 1995, A\&A, 300, 219

Tycner, C., Gilbreath, G. C., Zavala, R. T., Armstrong, J. T. et al. 2006, AJ, 131, 2710

Tycner, C., Jones, C. E., Sigut, T. A. A., Schmitt, H. R. et al. 2008, ApJ, 689, 461 